\title{
Psychosocial, behavioral, and supportive interventions for pediatric, adolescent, and young adult cancer survivors: A systematic review and meta-analysis
}

\author{
Anao Zhang ${ }^{\mathrm{a}, *}$, Kaipeng Wang ${ }^{\mathrm{b}}$, Bradley Zebrack ${ }^{\mathrm{a}}$, Chiu Yi Tan ${ }^{\mathrm{c}}$, Emily Walling ${ }^{\mathrm{d}}$, \\ Rashmi Chugh $^{\mathrm{e}}$
}

${ }^{a}$ University of Michigan School of Social Work, United States

${ }^{\mathrm{b}}$ University of Denver Graduate School of Social Work, United States

${ }^{\mathrm{c}}$ University of Michigan Medical School Department of Psychiatry, United States

${ }^{\mathrm{d}}$ University of Michigan Medical School Department of Pediatrics, United States

${ }^{\mathrm{e}}$ University of Michigan Medical School Department of Internal Medicine, United States

\section{A R T I C L E I N F O}

\section{Keywords:}

Pediatric cancer

Adolescent and young adult cancer Survivorship

Systematic review and meta-analysis

Psycho-oncology

\begin{abstract}
A B S T R A C T
Background: Pediatric, adolescent, and young adult (PAYA) cancer survivors suffer from multiple domains of adverse psychosocial and behavioral outcomes during and after their cancer treatment. This study conducted a systematic review and metaanalysis of psychosocial, behavioral, and supportive interventions for PAYA cancer survivors.

Methods: We searched 11 electronic databases, 4 professional websites, and manual search of reference lists in existing reviews. We selected randomized controlled trials and controlled trials without randomization focusing on PAYA cancer survivors across six outcome domains.

Results: We included 61 studies (4,402 participants) published between 1987 and 2020 . Overall risk of bias across studies was low. We identified an overall moderate and statistically significant treatment effect size for PAYA cancer survivors across six outcome domains.

Conclusion: psychosocial, behavioral, and supportive interventions were overall effective for PAYA cancer survivors. However, interventions were not effective for certain outcome domains, and less effective among AYA versus pediatric cancer survivors.
\end{abstract}

\section{Introduction}

Pediatric, adolescent, and young adult cancer survivors (PAYA survivors) are individuals who have/had a cancer diagnosis between the ages 0-39 years old. In 2018, there were over 650,000 PAYA survivors in the United States (Close et al., 2019; Segal et al., 2018). Despite recent medical advances and significant improvements in 5-year cancer survival rates, most PAYA survivors experience adverse late, long-term, or persistent medical, behavioral, psychosocial, spiritual, and/or existential outcomes cause by cancer or cancer treatments (Shay et al., 2017; Marjerrison and Barr, 2018; Hydeman et al., 2019). PAYA survivors are more likely than older cancer survivors to suffer from bio-psycho-social-spiritual challenges as a result of their cancer experience, such as risky health behaviors, low self-esteem, increased mortality, and suicide risk (Penn et al., 2017; Zhou et al., 2019; Harashima and Fujimori, 2020). Given their age, PAYA cancer survivors are arguably the most vulnerable cancer sub-population to adverse survivorship outcomes (Lang et al., 2018).

The known risks, complex needs, and challenging survivorship outcomes for PAYA survivors highlight the importance of understanding the efficacy of evidence-informed psychosocial, behavioral, and supportive interventions that health care providers may integrate into their practice. We broadly define psychosocial, behavioral, and supportive interventions as any type of interventions supporting PAYA cancer survivors that are not medical or pharmaceutical in nature (Page and Adler, 2008). These may include psycho-therapeutic interventions (like

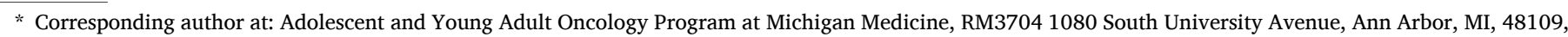
United States.

E-mail address: zhangan@umich.edu (A. Zhang).
} 
motivational interviewing or behavioral activation), (psycho)educational programs (to increase PAYA survivors' cancer-related knowledge), and online peer support groups to increase PAYA survivors' physical activities, among other forms of interventions.

Although systematic reviews and meta-analyses on this topic exist, (Braam et al., 2016; Bukola and Paula, 2017; Mizrahi et al., 2017; Pai et al., 2006; Richter et al., 2015) gaps in this literature warrant an updated systematic review and synthesis of the interventions examined in those studies. First, all existing systematic reviews focused on narrow topics, e.g. cognitive-behavioral therapy for depressive symptoms only, and thus do not reflect the complex nature of survivors' post-treatment experience and the heterogeneity of interventions for PAYA cancer survivors. It is important to be inclusive of all types of interventions for multiple domains of outcomes because oncology providers often need to make treatment recommendations for PAYA patients facing a multitude of bio-psycho-social-behavioral challenges. Drawing on several conceptual and theoretical papers on survivorship care among PAYA survivors, (Shay et al., 2017; Marjerrison and Barr, 2018; Hydeman et al., 2019; Baird et al., 2019; Lewis et al., 2014), authors of this review developed a 6-domain conceptual framework to guide the categorization of survivorship outcomes: 1 . (Physical) health domain, including physical symptoms and functional health, and health-related behaviors; 2. Psychological domain, including mental health, cognitive health, and other types of psychological outcomes; 3. Social (relational) domain; 4. Academic domain; 5. General quality of life; and 6. Cancer related knowledge (see eAppendix for detail).

Second, due to the narrow scope of many reviews (thus small number of included studies), most existing review studies were unable to perform meta-analysis to estimate an overall treatment effect. While still highly valuable, existing qualitative systematic reviews did not provide a statistical analysis on the treatment effect of different types of interventions, which makes it challenging for oncology providers to engage in evidence-based practice. Finally, many existing reviews were completed before 2015 (5 years ago). Therefore, an update of the literature is also warranted.

Given gaps in the existing literature, we conducted a systematic review and meta-analysis of clinical trials evaluating psychosocial and behavioral interventions for PAYA cancer survivors. The goal is to provide an updated, yet comprehensive review of all psychosocial interventions intended to have effects on the multiple and varied outcome domains of survivorship (defined above). Doing so will better inform survivorship care for this population, as it will provide a more nuanced and focused analyses to inform best practices for psychosocial oncology care providers. In this review, a cancer survivor is defined as any individual since the moment of receiving a cancer diagnosis (Mayer et al., 2017). Adapting such definition would enable this review including treatments supporting PAYA cancer survivors both during and after their cancer treatments.

\section{Methods}

The Methodological Expectations of Cochrane Intervention Reviews (MECIR) guided the conduct of this systematic review and metaanalysis, and findings are reported in accordance with the Preferred Reporting Items for Systematic Review and Meta-analysis (PRISMA). Study team included experts in pediatric, medical, and psychosocial oncology, including investigators with extensive experience in systematic review and meta-analysis. This review was pre-registered at PROSPERO: CRD42020200987.

\subsection{Search methods}

The study searched for controlled clinical trials (with or without randomization) of psychosocial and behavioral interventions for PAYA cancer survivors. We included all eligible articles from inception through June 1st, 2020. We carried out an electronic search across 11 databases, 4 professional websites, and a manual search of the reference lists from published reviews relating to our study (eSupplement1). Two of the authors (A.Z. and C.T.) independently screened all trials to determine eligibility first by titles and abstracts, then by full texts using the Cochrane recommended platform, Covidence. Disagreements were resolved first through consensus and further discussion with a third/ senior reviewer (B.Z.).

\subsection{Population, intervention, and outcome measures}

Controlled trials for cancer survivors who fit NCI's definition for pediatric, adolescent and young adult (diagnosed between the ages of 0-39 years) were eligible for inclusion. Any interventions that were solely medical or pharmaceutical were excluded from this review. The primary outcome meta-analyzed in this study was the survivorship outcome, operationalized by combining all bio-psycho-social-behavioralspiritual outcome domains together to reflect the complex nature of PAYA cancer survivorship. A second level of analysis involved examination of intervention effects separately by pre-determined domains of the survivorship (defined earlier): 1. (physical) health outcomes; 2. psychological outcomes; 3 . social outcomes; 4 . academic outcomes; 5. general quality of life; and 6 . cancer knowledge. We included studies published in English but posed no restriction for type of publication.

\subsection{Data extraction}

A data extraction sheet (eSupplement2) was developed by the study team including pediatric and adult clinicians as well as social and behavioral scientists in the psycho oncology realm. Two of us (A.Z. and C.T.) independently extracted the data from the included trials. In instances of disagreement, a third reviewer was consulted (B.Z.).

\subsection{Risk of Bias assessment}

Risk of bias was assessed with the Cochrane Collaboration's Risk of Bias tool 2nd version (RoB 2) (Sterne et al., 2019) for randomized controlled trial (RCT) and the Risk of Bias in Non-randomized Studies of Interventions (ROBINS-I) tool (Sterne et al., 2016) for controlled trial study without randomization. The RoB 2 tool is the recommended tool to assess for RCTs included in Cochrane reviews. The RoB 2 tool evaluates 5 domains of bias focusing on different aspects of RCTs, and provides researchers a structured way scoring individual RCTs. The 5 domains are: 1. Risk of bias arising from the randomization process; 2. Risk of bias due to deviations from the intended intervention; 3. (Risk of bias due to) Missing outcome data; 4. Risk of bias in measurement of the outcome; and 5. Risk of bias in selection of the reported result. The ROBINS-I tool is the Cochrane recommended tool to evaluate risk of bias for non-randomized studies of interventions. The ROBINS-I tool systematically evaluates 7 domains of bias including: 1 . Bias due to confounding; 2. Bias in selection of participants into the study; 3 . Bias in classification of interventions; 4. Bias due to deviations from intended interventions; 5 . Bias due to missing data; 6 . Bias in measurement of outcomes; and 7. Bias in selection of the reported result.

\subsection{Data synthesis and statistical analysis}

Analyses were performed with R Statistical Software (version 4.0.2) with the metafor and robumeta package, using robust variance estimation (RVE) in meta-regression (Hedges et al., 2010). All outcomes were continuous, and we calculated the between group small sample size corrected Hedges' $g$ effect size, noted as $d$ in this study, for meta-analysis. The sampling error variance of each $d$ was calculated using procedures recommended by Cooper and colleagues (Cooper et al., 2019).

Given the clustered nature of our data, i.e., including multiple effect sizes reported in a single study, we used meta-regression with RVE, 
which allows meta-analyzing multiple treatment effect sizes from the same study without compromising the validity of statistical inference. Heterogeneity was assessed using $\tau^{2}$ to estimate the between-study variance in study-average effect sizes. Because meta-regression with RVE produces robust estimation of the variance component regardless of the model selection (i.e. fixed versus random effect models), all metaanalyses were conducted without specifying a variance modeling strategy. We planned subgroup analyses for survivorship outcome based on important study, participant, and intervention characteristics, including: 1. study design (RCT versus controlled trial without randomization); 2. age of PAYA survivors (pediatric only, or AYA only, or both); 3. intervention modality (individual versus non-individual intervention); 4. treatment component (single component, like cognitive behavioral therapy, versus multiple components, like behavioral activation plus peer support); and 5. treatment formality (in-person versus tele-health interventions). Moderator analyses were conducted for the survivorship outcome overall (across domains) and for specific domains of survivorship outcomes. Sensitivity analyses were conducted accounting for risk of bias and outliers to ensure findings were not significantly altered. Publication bias was evaluated through both visual examination of funnel plots and the Vevea and Woods sensitivity weighting models (Vevea and Woods, 2005).

\section{Results}

\subsection{Literature search}

An initial search of literature identified 10,026 references from electronic database searches and 34 relevant systematic reviews and/or meta-analysis studies to search through reference lists (Fig. 1). Title and abstract screening resulted in 94 references for full text screening, which further retained 59 studies for data synthesis. A search through the reference list of 34 relevant systematic reviews and/or meta-analysis studies identified 2 additional eligible trials, resulting in a final sample size of 61 studies and 664 effect sizes for data synthesis. On average, each study reported 11 effect sizes, ranging from 1 to 38 effect sizes per study with a standard deviation of 8.47. It is important to re-iterate that meta-regression with RVE effectively accounts for the clustered nature of our data so that those studies with more effect sizes are not overweighted in the final meta-analytic results.

\subsection{Trial characteristics}

Fifty-three randomized controlled trials and eight controlled trials without randomization, published between 1987 and 2020, included a total of 4,402 PAYA cancer survivors. Mean age across studies was 15.38 years old, ranging from 6.5-32.79 years. Less than half of all participants ( $46.59 \%)$ were female. Forty-four trials reported an average of $54.16 \%$ of the participants being White, which included 9 clinical trials exclusively targeting non-White races. Most trials were conducted in North America, i.e. United States or Canada $(n=38)$, seven in Europe, six in Hong Kong, one in Taiwan, seven in middle East countries, and two were multi-nation trials. All but one were trials published in peer-reviewed academic journals.

Fifty-eight of the 61 trials (95.08\%) focused on PAYA survivors with various cancer diagnosis, leaving three diagnosis-specific trials one focusing on child leukemia, one on acute lymphoblastic leukemia, and one on medulloblastoma. Thirty-six trials (59.02\%) focused on pediatric cancer survivors, sixteen trials focused on AYA cancer survivors, leaving 9 trials focusing on both pediatric and AYA cancer survivors. Fifty-two trials $(85.25 \%)$ delivered individual-based interventions and nine trials (14.75\%) administered group-based or family-based interventions. Thirty-nine trials $(63.93 \%)$ delivered interventions with multiple components, e.g., psychoeducation plus skill training, and twenty-two trials $(36.07 \%)$ delivered single component interventions, e.g., cognitive behavioral therapy, digital story-telling, and cognitive remediation treatment. Twenty-three trials (37.70\%) evaluated psychoeducation and/or skill training interventions, twenty-one (34.43\%) evaluated psychotherapeutic interventions, and seventeen (27.87\%) trials involved supportive care interventions, e.g., distraction for chemotherapy distress or digital storytelling application to improve quality of life. Thirty-three trials delivered in person intervention and twenty-nine trials used technology-assisted intervention. Supplemental Table 1 provides details of the 61 studies including detailed references.

\subsection{Risk of Bias and publication Bias}

Risk of bias assessment (Supplemental TaTable 2ble 2) revealed overall low risk of bias for randomized controlled trials as well as for controlled trials without randomization. Several randomized controlled trials reported moderate risk of bias due to randomization and missing

\begin{tabular}{|c|c|}
\hline $\begin{array}{l}\text { Initial pool of studies from databases } \\
\text { after duplications removed }(n=10,026)\end{array}$ & $\begin{array}{l}\text { Manual search of professional websites } \\
\text { did not identify additional studies }\end{array}$ \\
\hline $\begin{array}{c}\text { A total of } 10,026 \text { studies for title and } \\
\text { abstract screening }\end{array}$ & $\begin{array}{l}\text { A total of 9,932 studies clearly did not } \\
\text { meet inclusion criteria, thus excluded }\end{array}$ \\
\hline $\begin{array}{l}\text { A total of } 94 \text { studies were screened for } \\
\text { full-text eligibility review }\end{array}$ & $\begin{array}{l}\text { We further excluded } 35 \text { studies for } \\
\text { specific reasons* }\end{array}$ \\
\hline $\begin{array}{l}\text { A total of } 59 \text { studies were eligible for } \\
\text { inclusion and meta-analysis }\end{array}$ & $\begin{array}{l}\text { Identified } 2 \text { additional trials from } \\
\text { existing review studies }\end{array}$ \\
\hline $\begin{array}{l}\text { A final sample size of } 61 \text { trials included } \\
\text { and analyzed in this study }\end{array}$ & \\
\hline
\end{tabular}

* Reasons for full-text exclusion: 1 . No control group $(\mathrm{n}=9)$; 2 . Included patients older than 39 years old $(n=4) ; 3$. Interventions focused on parents and parent related outcomes $(n=7)$; 4. Pharmacology interventions $(\mathrm{n}=3) ; 5$. Qualitative studies with no data available for meta-analysis $(\mathrm{n}=5)$; and 6. Other reasons $(\mathrm{n}=7)$

Fig. 1. PRISMA Diagram. 


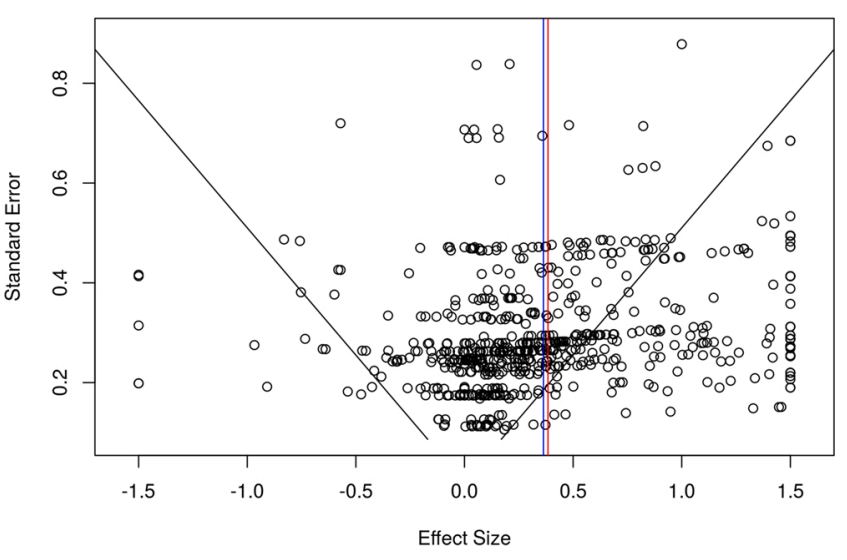

Fig. 2. Funnel Plot.

outcome data, whereas most controlled trials reported moderate risk of bias due to missing outcome data. Funnel plot indicated low concern for publication bias (Fig. 2).

\subsection{Survivorship outcomes}

Sixty-one trials of 664 effect sizes across the six pre-determined domains of survivorship outcome reported an overall statistically significant treatment effect, $d=0.470,95 \% C I 0.360,0.579, \tau^{2}=0.24$. Subgroup analyses by study and intervention characteristics (Table 1) revealed that the treatment effect was statistically significant regardless of study design (RCT versus controlled trials without randomization),

Table 1

Overall treatment effect and subgroup analysis results by study and intervention characteristics*.

\begin{tabular}{|c|c|c|c|c|c|}
\hline & Estimate & df & $\mathrm{N} / \mathrm{K}$ & $95 \%$ CI & $\mathrm{p}$ value \\
\hline Overall Effect & 0.470 & 57.2 & $\begin{array}{l}61 / \\
664\end{array}$ & $\begin{array}{l}{[0.360} \\
0.579]\end{array}$ & $\begin{array}{l}< \\
0.0001\end{array}$ \\
\hline \multicolumn{6}{|l|}{ Study Design** } \\
\hline - RCT & 0.449 & 49.6 & $\begin{array}{l}53 / \\
577\end{array}$ & $\begin{array}{l}{[0.336} \\
0.563]\end{array}$ & $\begin{array}{l}< \\
0.0001\end{array}$ \\
\hline $\begin{array}{l}\text { - CT without } \\
\text { randomization }\end{array}$ & 0.608 & 6.69 & $8 / 87$ & $\begin{array}{l}{[0.146,} \\
1.070]\end{array}$ & $\begin{array}{l}= \\
0.0174\end{array}$ \\
\hline \multicolumn{6}{|l|}{ Targeted Population $* * *$} \\
\hline - Pediatric only & 0.573 & 34.1 & $\begin{array}{l}36 / \\
302\end{array}$ & $\begin{array}{l}{[0.405} \\
0.741]\end{array}$ & $\begin{array}{l}< \\
0.0001\end{array}$ \\
\hline - AYA only & 0.211 & 11.7 & $\begin{array}{l}16 / \\
205\end{array}$ & $\begin{array}{l}{[0.102} \\
0.320]\end{array}$ & $\begin{array}{l}= \\
0.0013\end{array}$ \\
\hline - Pediatric and AYA & 0.540 & 7.83 & 9/157 & $\begin{array}{l}{[0.311,} \\
0.769]\end{array}$ & $\begin{array}{l}= \\
0.0006\end{array}$ \\
\hline \multicolumn{6}{|l|}{ Treatment Modality } \\
\hline - Individual-based & 0.452 & 48.5 & $\begin{array}{l}52 / \\
600\end{array}$ & $\begin{array}{l}{[0.326} \\
0.578]\end{array}$ & $\begin{array}{l}< \\
0.0001\end{array}$ \\
\hline - Other modalities & 0.551 & 7.87 & $9 / 64$ & $\begin{array}{l}{[0.313,} \\
0.790]\end{array}$ & $\begin{array}{l}= \\
0.0007\end{array}$ \\
\hline \multicolumn{6}{|l|}{ Treatment Component } \\
\hline - Single component & 0.380 & 20.2 & $\begin{array}{l}22 / \\
312\end{array}$ & $\begin{array}{l}{[0.220} \\
0.540]\end{array}$ & $\begin{array}{l}< \\
0.0001\end{array}$ \\
\hline - Multiple components & 0.520 & 36.0 & $\begin{array}{l}39 / \\
352\end{array}$ & $\begin{array}{l}{[0.370} \\
0.655]\end{array}$ & $\begin{array}{l}< \\
0.0001\end{array}$ \\
\hline \multicolumn{6}{|l|}{ Formality } \\
\hline - In person treatment & 0.519 & 31.3 & $\begin{array}{l}33 / \\
377\end{array}$ & $\begin{array}{l}{[0.363,} \\
0.674]\end{array}$ & $\begin{array}{l}< \\
0.0001\end{array}$ \\
\hline - Telehealth involved & 0.407 & 24.5 & $\begin{array}{l}28 / \\
287\end{array}$ & $\begin{array}{l}{[0.244} \\
0.570]\end{array}$ & $\begin{array}{l}< \\
0.0001\end{array}$ \\
\hline
\end{tabular}

* $\boldsymbol{d} \boldsymbol{f}=$ degrees of freedom, it should be noted that for any effect size with a df $<$ 4 , a more conservative use of the $\mathrm{p}$ value should be adopted, i.e., $\mathrm{p}<0.01$ to be considered statistically significant. $\boldsymbol{N} / \boldsymbol{K}$ for which $\mathrm{N}$ stands for the number of study and $\mathrm{K}$ stands for the number of effect size estimates.

${ }^{* *}$ RCT $=$ randomized controlled trial; CT without randomization $=$ controlled trial without randomization.

${ }_{* * *}^{*}$ AYA $=$ adolescent and young adult.
Table 2

Univariate moderator analysis for survivorship outcomes*.

\begin{tabular}{llllll}
\hline & Coefficient & $\mathrm{df}$ & $\mathrm{N} / \mathrm{K}$ & $95 \% \mathrm{CI}$ & $\mathrm{p}$ value \\
\hline Age & -0.019 & 9.17 & $60 /$ & {$[-0.031$,} & $=$ \\
& & & 633 & $-0.007]$ & 0.0057 \\
AYA versus PAYA & -0.307 & \multirow{2}{*}{16.33} & $61 /$ & {$[-0.545$,} & $=$ \\
$* *$ & & & 664 & $-0.068]$ & 0.0149 \\
AYA versus & -0.329 & 28.4 & $61 /$ & {$[-0.533$,} & $=$ \\
Pediatric ${ }^{* * *}$ & & & 664 & $-0.124]$ & 0.0027 \\
\hline
\end{tabular}

* All subgroup variables were tested as moderators and only significant results were presented.

** An overall treatment effect for AYA survivors only when compared with an overall treatment effect for both pediatric and AYA survivors.

${ }_{* * *}$ An overall treatment effect for AYA survivors only when compared with an overall treatment effect for pediatric survivors only.

across targeted population (pediatric survivors only, or AYA survivor only, or PAYA survivors), treatment modalities (individual or nonindividual based intervention), treatment components (single or multiple components), and delivery format (in person delivery or telehealth involved).

Univariate moderator analyses revealed that age and targeted population were two significant moderators for survivorship outcomes (Table 2). Age significantly moderated the effect size of survivorship outcomes, $b=-0.019,95 \% C I-0.031,-0.007$. For each one-year increase in a study participants' average age, that study is expected to report 0.019 decrease in effect size. Studies targeting AYA survivors on average reported significantly smaller treatment effect than studies targeting PAYA survivors and pediatric survivors, $b=-0.307,95 \% C I-0.545$, -0.068 and $b=-0.329,95 \% C I-0.533,-0.124$, respectively. Outcome domain did not significantly moderate survivorship outcomes, meaning the difference of treatment effect between domains of survivorship outcomes did not significantly differ from zero.

\subsection{Domains of survivorship outcomes}

\subsection{1. (Physical) health outcomes}

Subgroup analyses and within subgroup moderator analyses across outcome domains were presented in Table 3 and Table 4. Thirty-eight

Table 3

Subgroup analysis results by domains of outcomes*.

\begin{tabular}{|c|c|c|c|c|c|}
\hline & Estimate & $\mathrm{df}$ & $\mathrm{N} / \mathrm{K}$ & $95 \%$ CI & $\mathrm{p}$ value \\
\hline Physical related outcomes & 0.424 & 35.8 & $\begin{array}{l}38 / \\
276\end{array}$ & $\begin{array}{l}{[0.260} \\
0.589]\end{array}$ & $\begin{array}{l}< \\
0.0001\end{array}$ \\
\hline - physical symptoms & 0.470 & 28.5 & $\begin{array}{l}31 / \\
178\end{array}$ & $\begin{array}{l}{[0.291,} \\
0.649]\end{array}$ & $\begin{array}{l}< \\
0.0001\end{array}$ \\
\hline - health behaviors & 0.421 & 17.8 & $\begin{array}{l}19 / \\
98\end{array}$ & $\begin{array}{l}{[0.141} \\
0.700]\end{array}$ & $\begin{array}{l}= \\
0.0054\end{array}$ \\
\hline $\begin{array}{l}\text { Psychological related } \\
\text { outcomes }\end{array}$ & 0.528 & 35.9 & $\begin{array}{l}39 / \\
262\end{array}$ & $\begin{array}{l}{[0.383} \\
0.674]\end{array}$ & $\begin{array}{l}< \\
0.0001\end{array}$ \\
\hline - mental health & 0.582 & 25.9 & $\begin{array}{l}29 / \\
99\end{array}$ & $\begin{array}{l}{[0.407} \\
0.757]\end{array}$ & $\begin{array}{l}< \\
0.0001\end{array}$ \\
\hline - cognitive wellness & 0.367 & 4.83 & $6 / 34$ & $\begin{array}{l}{[-0.192} \\
0.926]\end{array}$ & $\begin{array}{l}= \\
0.1510\end{array}$ \\
\hline - psychological wellness & 0.460 & 18.4 & $\begin{array}{l}20 / \\
129\end{array}$ & $\begin{array}{l}{[0.197} \\
0.723]\end{array}$ & $\begin{array}{l}= \\
0.0017\end{array}$ \\
\hline Social related outcomes & 0.219 & 11.3 & $\begin{array}{l}13 / \\
32\end{array}$ & $\begin{array}{l}{[0.051} \\
0.387]\end{array}$ & $\begin{array}{l}= \\
0.0153\end{array}$ \\
\hline $\begin{array}{l}\text { Academic related } \\
\text { outcomes }\end{array}$ & 0.257 & 8.63 & $\begin{array}{l}10 / \\
39\end{array}$ & $\begin{array}{l}{[-0.001} \\
0.515]\end{array}$ & $\begin{array}{l}= \\
0.0508\end{array}$ \\
\hline $\begin{array}{l}\text { Cancer knowledge related } \\
\text { outcomes }\end{array}$ & 0.195 & 4.07 & $6 / 19$ & $\begin{array}{l}{[-0.152} \\
0.543]\end{array}$ & $\begin{array}{l}= \\
0.1940\end{array}$ \\
\hline General quality of life & 0.417 & 15.2 & $\begin{array}{l}17 / \\
36\end{array}$ & $\begin{array}{l}{[0.193,} \\
0.642]\end{array}$ & $\begin{array}{l}= \\
0.0012\end{array}$ \\
\hline
\end{tabular}

* $\boldsymbol{d} \boldsymbol{f}=$ degrees of freedom, it should be noted that for any effect size with a $\mathrm{df}<$ 4 , a more conservative use of the $\mathrm{p}$ value should be adopted, i.e., $\mathrm{p}<0.01$ to be considered statistically significant. $\boldsymbol{N} / \boldsymbol{K}$ for which $\mathrm{N}$ stands for the number of study and $\mathrm{K}$ stands for the number of effect size estimates. 
Table 4

Univariate moderator analysis for subdomains of outcomes*.

\begin{tabular}{|c|c|c|c|c|c|}
\hline & Estimate & df & $\mathrm{N} / \mathrm{K}$ & $95 \% \mathrm{CI}$ & $\mathrm{p}$ value \\
\hline \multicolumn{6}{|l|}{ Physical Outcomes } \\
\hline Age & -0.018 & 7.71 & $\begin{array}{l}38 / \\
276\end{array}$ & $\begin{array}{l}{[-0.033} \\
-0.003]\end{array}$ & $\begin{array}{l}= \\
0.0245\end{array}$ \\
\hline \multicolumn{6}{|l|}{ Psychological Outcomes } \\
\hline Age & -0.026 & 6.17 & $\begin{array}{l}37 / \\
254\end{array}$ & $\begin{array}{l}{[-0.042,} \\
-0.010]\end{array}$ & $\begin{array}{l}= \\
0.0068\end{array}$ \\
\hline $\begin{array}{l}\text { Non-individual vs } \\
\text { Individual based }\end{array}$ & 0.324 & 7.02 & $\begin{array}{l}39 / \\
262\end{array}$ & $\begin{array}{l}{[0.053,} \\
0.595]\end{array}$ & $\begin{array}{l}= \\
0.0256\end{array}$ \\
\hline AYA versus PAYA** & -0.332 & 14.71 & $\begin{array}{l}39 / \\
262\end{array}$ & $\begin{array}{l}{[-0.590} \\
-0.073]\end{array}$ & $\begin{array}{l}= \\
0.0154\end{array}$ \\
\hline AYA versus Pediatric ${ }^{* * *}$ & -0.360 & 19.1 & $\begin{array}{l}39 / \\
262\end{array}$ & $\begin{array}{l}{[-0.659} \\
-0.060]\end{array}$ & $\begin{array}{l}= \\
0.0211\end{array}$ \\
\hline \multicolumn{6}{|l|}{$\begin{array}{l}\text { Quality of Life } \\
\text { Outcomes }\end{array}$} \\
\hline Age & -0.133 & 6.55 & $\begin{array}{l}17 / \\
36\end{array}$ & $\begin{array}{l}{[-2.40} \\
-0.025]\end{array}$ & $\begin{array}{l}= \\
0.0226\end{array}$ \\
\hline
\end{tabular}

* All subgroup variables were tested as moderators and only significant results were presented.

${ }^{* *}$ An overall treatment effect for AYA survivors only when compared with an overall treatment effect for both pediatric and AYA survivors.

${ }_{* * * *}$ An overall treatment effect for AYA survivors only when compared with an overall treatment effect for pediatric survivors only.

studies including 276 effect sizes reported an overall statistically significant treatment effect, $d=0.424,95 \%$ CI $0.260,0.589, \tau^{2}=0.33$. Physical health outcomes were further categorized into 1 . physical symptom outcomes and 2 . health behavior outcomes. Physical symptom outcomes (31 studies and 178 effect sizes) reported an average treatment effect $d=0.470,95 \% C I 0.291,0.649, \tau^{2}=0.26$. Health behavior outcomes (19 studies and 98 effect sizes) reported an average treatment effect $d=0.421,95 \%$ CI 0.141, 0.700, $\tau^{2}=0.39$.

Age significantly moderated physical outcomes among PAYA cancer survivors, $b=-0.018,95 \% C I-0.033,-0.003$. For each one-year increase in a study participants' average age, that study is expected to report 0.018 decrease in effect size. No other study, participant, and intervention characteristics were significant moderators for physical health outcomes.

\subsubsection{Psychological outcomes}

Thirty-nine studies including 262 effect sizes reported an overall statistically significant treatment effect, $d=0.528,95 \%$ CI 0.383 , $0.674, \tau^{2}=0.19$. Psychological outcomes were further categorized into 1. mental health outcomes, 2. cognitive wellness outcomes, and 3. general psychological wellness outcomes. Mental health outcomes (29 studies and 99 effect sizes) reported an average treatment effect $d=$ 0.582, $95 \% \mathrm{CI}, 0.407,0.757, \tau^{2}=0.18$. Cognitive wellness outcomes (6 studies and 34 effect sizes) reported an average non-significant treatment effect $d=0.367,95 \% C I-0.192,0.926, \tau^{2}=0.21$. General psychological wellness (20 studies and 129 effect sizes) reported an average treatment effect $d=0.460,95 \%$ CI 0.183, 0.691, $\tau^{2}=0.27$.

Moderator analyses revealed that age, intervention modality (individual versus non-individual), and targeted population significantly moderated PAYA survivors' psychological outcomes. For each one-year increase in a study participants' average age, that study on average is expected to report a decrease in treatment effect of $0.026, b=-0.026,95$ $\%$ CI, -0.042, -0.010. Non-individual interventions (e.g., family-based or peer-supported) reported significantly greater treatment effect than individual-based interventions, $b=0.324,95 \%$ CI 0.053, 0.595. Studies targeting AYA survivors reported an average of 0.332 lower treatment effect than studies targeting PAYA survivors, $b=-0.332$, $95 \% C I-0.590$, -0.073 , as well as an average of 0.360 lower treatment effect than studies targeting pediatric survivors, $b=-0.360,95 \% C I-0.659,-0.060$.

\subsubsection{Social and academic outcomes}

Thirteen studies including 32 effect sizes of social outcomes reported an overall statistically significant treatment effect, $d=0.219,95 \% C I$ $0.046,0.359, \tau^{2}=0.05$. Ten studies including 39 effect sizes of academic outcomes reported an overall statistically non-significant treatment effect, $d=0.257,95 \% \mathrm{CI},-0.001,0.515, \tau^{2}=0.09$.

None of the study, participant, and intervention characteristics significantly moderated treatment effect size for social or academic outcomes.

\subsection{Cancer knowledge outcomes and general quality of life}

Six studies including 19 effect sizes of cancer knowledge outcomes reported an overall statistically non-significant treatment effect, $d=$ $0.195,95 \% C I-0.152,0.543, \tau^{2}=0.06$. Seventeen studies including 36 effect sizes of general quality of life reported an overall statistically significant treatment effect, $d=0.417,95 \%$ CI 0.193, 0.642, $\tau^{2}=0.14$.

Age was the only significant moderator for quality of life outcomes, $b=-0.133,95 \% \mathrm{CI},-2.40,-0.025$. For each year increase in a study participants' average age, a study is expected to report 0.133 lower treatment effect.

\section{Discussion}

This systematic review and meta-analysis evaluated psychosocial, behavioral, and supportive interventions for PAYA cancer survivors across multiple and varied survivorship outcome domains, and when delivered at various points in time throughout a continuum of cancer care. Overall, this study identified a moderate treatment effect for interventions intended to affect survivorship outcomes, with the greatest treatment effect sizes observed among psychological and physical domains, and general quality of life. More specifically, the two strongest treatment effects were observed among mental health outcomes and physical symptoms related to cancer treatment. Such findings were overall positive because compromised mental health and physical side effects are amongst the top cited short- and long-term adverse outcomes related to cancer treatment.

Although statistically significant, existing interventions' overall treatment effect for social (relational) outcomes was small. This is concerning because socialization is critical for achieving multiple milestones during the PAYA age range. Furthermore, the overall treatment effects for academic and cancer knowledge outcomes were statistically non-significant, highlighting the importance of more research into these domains. Most interventions targeting PAYA survivors' academic or cancer knowledge outcomes were (psycho)educational programs that can be didactic and text heavy. As a result, PAYA cancer survivors may have low engagement with these interventions, resulting in low treatment effect. This highlights the importance of future research on developing and implementing engaging and interactive (psycho)educational programs to improve PAYA cancer survivors' academic and cancer knowledge outcomes.

A notable finding of this study is that treatment effects for survivorship outcomes differ by age/developmental stages within the PAYA age range. In comparison to pediatric cancer survivors, treatment effects for multiple domains of survivorship outcomes were significantly lower among AYA cancer survivors. Despite recent national attention to survivorship needs of AYA cancer survivors, psychosocial interventions remain inadequate for this population, which calls for persistent clinical and research effort to improve care and survivorship outcomes for this unique population of cancer survivors. Cancer survivors who are adolescents or emerging adults (15-26 years) receiving care at pediatric hospitals often find their age-specific needs are not sufficiently met (D'Agostino et al., 2011; Wong et al., 2017) given many pediatric hospitals (and programs offered) are designed for young children. This could explain the treatment effect difference between pediatric versus 
AYA cancer survivors. Another possible explanation could be the wide age spectrum of the AYA survivor population in comparison to pediatric cancer survivors. Most interventions for pediatric cancer survivors target individuals between 5-13 years old whereas AYA is defined between 15-39 years old. Although many researchers have agreed that this age range includes three distinct developmental stages (Barnett et al., 2016; Zhang et al., 2020), i.e. adolescence (15-17), emerging adulthood (18-25), and young adulthood (26-39), many psychosocial research on AYA focused on all age stages to increase participants' sample size and study feasibility. As a result, tested interventions may report lower treatment effect when compared with interventions developed targeting a smaller age range, e.g. young adult cancer survivors 18-25 years old.

In-person and telehealth-involved psychosocial interventions for PAYA cancer survivors were equally effective. Although in-person interventions reported greater treatment effect $(d=0.519)$ than telehealth-involved interventions $(d=0.397)$, the difference was not statistically significant. While encouraging, such finding does enunciate the significant needs of telehealth and tele-mental health research to improve survivorship outcomes for AYA cancer survivors, especially for the fact that AYA cancer survivors are a tech-prone and savvy generation.

\subsection{Findings in relation with current knowledge and existing reviews}

Our literature search identified 5 existing meta-analysis studies including one focusing on physical exercise training for PAYA cancer survivors' outcomes (Braam et al., 2016) $(n=6)$; one focusing on psychosocial intervention for AYA cancer survivors' outcomes (Richter et al., 2015) ( $\mathrm{n}=7)$ and three focusing on pediatric cancer survivors (Bukola and Paula, 2017; Mizrahi et al., 2017; Pai et al., 2006). Our findings were consistent with existing reviews evaluating specific types of intervention for a subdomain of outcome, e.g., physical exercise for health outcomes among childhood cancer survivors (Mizrahi et al., 2017). However, all existing reviews reported small treatment effects, which suggests limited clinical significance to these findings. To our knowledge, this review has a comprehensive and inclusive scope ( $\mathrm{n}=$ 61, including 664 effect sizes and 4402 PAYA cancer survivors) evaluating psychosocial interventions for PAYA survivors' outcomes. We also provided subgroup and moderator analyses that allowed us to evaluate outcome domains that were not previously synthesized quantitatively, i. e., academic outcomes, and cancer knowledge, to provide better guidance to the psychosocial care for PAYA survivors.

\subsection{Clinical implications}

Both pediatric and AYA cancer survivors are likely to benefit from psychosocial interventions for physical, psychological, social-relational outcomes and quality of life, but not for academic related outcomes or to improve their cancer knowledge. Recommendations for psychosocial interventions need to account for a patient's age as the strongest effects were observed among studies for pediatric survivors. Especially for psychological outcomes, oncology providers need to be aware that existing research have reported significantly greater effect for pediatric cancer survivors than their AYA counterparts.

\subsection{Strengths and limitations}

This review was conducted and reported following established methodological guidelines. We used an a priori defined protocol and carried out an extensive literature search including both scientific and gray literature. Our focus on survivorship as a higher order construct is inclusive of common bio-psycho-social-spiritual challenges faced by PAYA cancer survivors. We used advanced statistical methods to overcome known challenges of meta-analysis, i.e., including multiple measures of the same construct from the same study, to optimize statistical power. Similarly, the large sample size of included clinical trials and effect sizes alongside an overall strong quality rating of included studies provide us confidence in interpreting study findings.

This review was limited by the available data, which were sometimes insufficient to conduct more complex analysis, i.e., multiple moderator analysis in meta-regression. Besides, many studies included in this review did not clearly distinguish interventions for delivery during treatment from those intended to post-treatment survivors, which prevented us from statistically comparing interventions at different care timepoints. In addition, there is always a chance that we did not include all available trials, though it is unlikely to impact our overall findings. Finally, due to limited space, we did not report further subgroup and moderator analysis within each domains of outcomes, which should be further disseminated in future publications.

\subsection{Conclusion}

In this systematic review and meta-analysis of psychosocial intervention for PAYA cancer survivors' survivorship outcomes, we identified an overall moderate statistically significant treatment effect. We found existing psycho-oncology interventions effective for physical, psychological, social, and quality of life outcomes but not for academic outcomes and cancer related knowledge. We are fairly confident with our findings given the scope of this review and the quality of included studies in general. Additional analyses focusing on subdomains of outcomes should be reported in subsequent publications.

\section{Declaration of Competing Interest}

The authors report no declarations of interest.

\section{Acknowledgement}

A. Zhang received research support from the University of Michigan Vivian A. (U070401) and James L. Curtis School of Social Work Center for Health Equity Research and Training, Signature Programs Initiatives.

\section{Appendix A. Supplementary data}

Supplementary material related to this article can be found, in the online version, at doi:https://doi.org/10.1016/j.critrevonc.2021.10 3291.

\section{References}

Baird, H., Patterson, P., Medlow, S., Allison, K.R., 2019. Understanding and improving survivorship care for adolescents and young adults with cancer. J. Adolesc. Young Adult Oncol. 8 (5), 581-586.

Barnett, M., McDonnell, G., DeRosa, A., Schuler, T., Philip, E., Peterson, L., et al., 2016. Psychosocial outcomes and interventions among cancer survivors diagnosed during adolescence and young adulthood (AYA): a systematic review. J. Cancer Surviv. 10 (5), 814-831.

Braam, K.I., van der Torre, P., Takken, T., Veening, M.A., van Dulmen-den Broeder, E., Kaspers, G.J., 2016. Physical exercise training interventions for children and young adults during and after treatment for childhood cancer (Review). Cochrane Database Syst. Rev. 3 .

Bukola, I.M., Paula, D., 2017. The effectiveness of distraction as procedural pain management technique in pediatric oncology patients: a meta-analysis and systematic review. J. Pain Symptom Manag. 54 (4), 589-600.

Close, A.G., Dreyzin, A., Miller, K.D., Seynnaeve, B.K., Rapkin, L.B., 2019. Adolescent and young adult oncology-past, present, and future. CA Cancer J. Clin. 69 (6), 485-496.

Cooper, H., Hedges, L.V., Valentine, J.C., 2019. The Handbook of Research Synthesis and Meta-analysis. Russell Sage Foundation.

D’Agostino, N.M., Penney, A., Zebrack, B., 2011. Providing developmentally appropriate psychosocial care to adolescent and young adult cancer survivors. Cancer-Am Cancer Soc. 117 (S10), 2329-2334.

Harashima, S., Fujimori, M., 2020. Risk of suicide among adolescents and young adults with cancer and a need for targeted interventions. Ann. Transl. Med. 8 (7), 428.

Hedges, L.V., Tipton, E., Johnson, M.C., 2010. Robust variance estimation in metaregression with dependent effect size estimates. Res. Synth. Methods 1 (1), 39-65. 
Hydeman, J.A., Uwazurike, O.C., Adeyemi, E.I., Beaupin, L.K., 2019. Survivorship needs of adolescent and young adult cancer survivors: a concept mapping analysis. J. Cancer Surviv. 13 (1), 34-42.

Lang, M.J., Giese-Davis, J., Patton, S.B., Campbell, D.J., 2018. Does age matter? Comparing post-treatment psychosocial outcomes in young adult and older adult cancer survivors with their cancer-free peers. Psycho-Oncology. 27 (5), 1404-1411.

Lewis, D.R., Seibel, N.L., Smith, A.W., Stedman, M.R., 2014. Adolescent and young adult cancer survival. J. Natl. Cancer Inst. Monographs 2014 (9), 228-235.

Marjerrison, S., Barr, R.D., 2018. Unmet survivorship care needs of adolescent and young adult cancer survivors. JAMA Network Open. 1 (2) e180350-e180350.

Mayer, D.K., Nasso, S.F., Earp, J.A., 2017. Defining cancer survivors, their needs, and perspectives on survivorship health care in the USA. Lancet Oncol. 18 (1), e11-e18.

Mizrahi, D., Wakefield, C.E., Fardell, J.E., Quinn, V.F., Lim, Q., Clifford, B.K., et al., 2017. Distance-delivered physical activity interventions for childhood cancer survivors: a systematic review and meta-analysis. Crit. Rev. Oncol. Hematol. 118, 27-41.

Page, A.E., Adler, N.E., 2008. Cancer Care for the Whole Patient: Meeting Psychosocial Health Needs. National Academies Press.

Pai, A.L., Drotar, D., Zebracki, K., Moore, M., Youngstrom, E., 2006. A meta-analysis of the effects of psychological interventions in pediatric oncology on outcomes of psychological distress and adjustment. J. Pediatr. Psychol. 31 (9), 978-988.

Penn, A., Kuperberg, A., Zebrack, B.J., 2017. Psychosocial issues in adolescent and Young adult patients and survivors. In: Bleyer, A., Barr, R., Ries, L., Whelan, J., Ferrari, A. (Eds.), Cancer in Adolescents and Young Adults, pp. 583-602.

Richter, D., Koehler, M., Friedrich, M., Hilgendorf, I., Mehnert, A., Weißflog, G., 2015. Psychosocial interventions for adolescents and young adult cancer patients: a systematic review and meta-analysis. Crit. Rev. Oncol. Hematol. 95 (3), 370-386.

Segal, R., Miller, K., Jemal, A., 2018. Cancer statistics. CA Cancer J. Clin. 68 (1), 7-30.

Shay, L.A., Parsons, H.M., Vernon, S.W., 2017. Survivorship care planning and unmet information and service needs among adolescent and young adult cancer survivors. J. Adolesc. Young Adult Oncol. 6 (2), 327-332.

Sterne, J.A., Hernán, M.A., Reeves, B.C., Savović, J., Berkman, N.D., Viswanathan, M., et al., 2016. ROBINS-I: a tool for assessing risk of bias in non-randomised studies of interventions. BMJ. 355

Sterne, J.A., Savović, J., Page, M.J., Elbers, R.G., Blencowe, N.S., Boutron, I., et al., 2019. RoB 2: a revised tool for assessing risk of bias in randomised trials. BMJ. 366.

Vevea, J.L., Woods, C.M., 2005. Publication bias in research synthesis: sensitivity analysis using a priori weight functions. Psychol. Methods 10 (4), 428.

Wong, A.W., Chang, T.T., Christopher, K., Lau, S.C., Beaupin, L.K., Love, B., et al., 2017 Patterns of unmet needs in adolescent and young adult (AYA) cancer survivors: in their own words. J. Cancer Surviv. 11 (6), 751-764.
Zhang, A., Hu, R., Wang, K., Antalis, E.P., 2020. Age moderates the association between psychological distress and engagement in mindfulness among cancer patients and survivors: a population-based study. J. Psychosoc. Oncol. 38 (5), 513-526.

Zhou, H., Xian, W., Zhang, Y., Yang, Y., Fang, W., Liu, J., et al., 2019. Suicide among cancer patients: adolescents and young adult (AYA) versus all-age patients. Ann. Transl. Med. 7 (22), 658.

Anao Zhang is Assistant Professor at the University of Michigan School of Social Work and Research Director for Clinical Services and Patient Outcomes at the Michigan Medicine Adolescent and Young Adult (AYA) Oncology Program. Dr. Zhang's research focuses on advancing the psychosocial care for AYA cancer survivors.

Kaipeng Wang is Assistant Professor at the University of Denver Graduate School of Social Work. Dr. Wang is interested social determinants of health and health disparity research, especially for older adults. Dr. Wang is also an applied methodologist with expertise in research design and data analysis.

Bradley Zebrack is Professor of Social Work at the University of Michigan and the Research Director at the Michigan Medicine Adolescent and Young Adult (AYA) Oncology Program. Dr. Zebrack's interests are in the area of health, medicine, and quality of life, with a primary interest in the effects of cancer on the psychosocial growth and development of AYA cancer survivors.

Chiu Yi Tan is a Research Coordinator at Michigan Medicine's Department of Psychiatry. Ms. Tan has a primary research interest in supporting AYA cancer survivors' psychological outcomes through developing and implementing psycho-therapeutic interventions.

Emily Walling is a Pediatric Oncologist and Co-Medical Director at the Michigan Medicine Adolescent and Young Adult (AYA) Oncology Program. Dr. Walling is interested in the impact of healthcare disparities on childhood and adolescent cancer outcomes. She is also interested in survivorship outcomes among those treated for cancer as a child or adolescent.

Rashmi Chugh is a Medical Oncologist and Co-Medical Director at the Michigan Medicine Adolescent and Young Adult (AYA) Oncology Program. Dr. Chugh is interested in clinical trials of investigational agents or novel combinations in sarcoma patients, as well as psychosocial care for AYA cancer survivors. 